\title{
Yield and Yield Components of Some Sugar Cane Varieties (Saccharum officinarum L.) as affected by Different Nitrogen Fertilization Levels Abd El-Azez, Y. M. ${ }^{1}$; S. R. Nagib ${ }^{2}$ and A. M. Elwan ${ }^{1}$ \\ ${ }^{1}$ Sugar crop dept, Agric, Rese, Center, Mallawy, Minia, Egypt. \\ ${ }^{2}$ Agron. Dept, Fac. Agric, Minia Univ. Minia, Egypt.
}

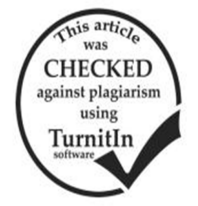

ABSTRACT

Two field trials were conducted during two successive growing seasons of 2016/2017 and 2017/2018 at Mallawy Agricultural Research Station, Agricultural Research Center, El-Minia Governorate (Middle Egypt) to study the effect of different levels of Nfertilization (160,190 and $220 \mathrm{~kg} / \mathrm{fed})$ on yield, yield components and quality of five sugar cane varieties (G.T.54-9(C9), G.2003-47, G.99-103, G.2004-27 and G.2010-7). A split plot design with three replications was used, main plots were represented by five sugar cane varieties (V1= G.T.54-9(C9), V2= G.2003-47, V3= G.99-103, V4= G.2004-27, V5= G.2010-7). The subplots were used for the three nitrogen levels $(160,190$ and $220 \mathrm{~kg} \mathrm{~N} /$ fed). The results indicated that: 1- Sugar cane varieties and N-fertilization levels exhibit significant effect on all studied traits in both seasons. Promising variety G.99-103 recorded the highest values of stalk hieght, stalk diameter $(\mathrm{cm})$, millable cane ton/fed and sugar yield (ton/fed) in both seasons, while G.T.54-9(C9) variety recorded the highest values of sucrose $\%$, purity $\%$ and sugar recovery $\%$, whoever G.2004-27 variety outyielded the highest value of TSS $\% 2$ - Increasing applied N levels from 160 up to $220 \mathrm{Kg} \mathrm{N} /$ fed significantly increased stalk height, stalk diameter(cm), millable cane ton / fed, sugar yield (ton/fed) and TSS (\%), while $190 \mathrm{Kg} \mathrm{N} /$ fed had the highest values of purity\% and Sugar recovery\%.3- The effect of interaction between sugar cane varieties and nitrogen levels on the studied traits was significant for stalk height in both seasons and purity\%, sucrose \% and T.S.S $\%$ in plant crop season only. Generally, it could be concluded that under Middle Egypt conditions, it is recommended to inoculate G.99103 sugar cane varieties with $220 \mathrm{Kg} \mathrm{N} /$ fed to maximize the productivity and quality of sugar cane yield.

Keywords: Sugar cane, Saccharum officinarum L., varieties, nitrogen fertilization levels, yield, quality.

\section{INTRODUCTION}

Sugar cane (Saccharum officinarum L.) requires substantial inputs of nitrogen to achieve maximum yields. Nitrogen is the most essential element having direct effect on cane growth, sugar cane yield, and juice quality. Studies have established that $\mathrm{N}$ - increase the quantity of green tops, yield components and yield of cane and sugar Azzazy and El-Geddawy, (2003); El-Geddawy et al. (2003); ElGeddawy et al. (2005) and Nassar et al. (2005) Similarly, Yousef et al. (2000) reported that nitrogen has significant influence on cane growth, yield, quality and recoverable sugar. However, nitrogen application at high rates exceeding sugar cane plant utilization has adverse effect on cane quality. Vinicius et al. (2018) provided that sugar cane varieties showed similar $\mathrm{N}$ requirements in the three evaluation cycles, on average $1.35 \mathrm{~kg} \mathrm{~N} /$ ton of cane in the cane-plant, 0.87 and $0.73 \mathrm{~kg} \mathrm{t}-1$ in the first and second regrowth, respectively, The nutrient constitutes less than $1 \%$ of sugar cane biomass, but its deficiency causes a reduction in the synthesis of chlorophyll, essential amino acids and the energy required to produce carbohydrates and carbon skeletons, directly reflecting the development and yield of the crop

However, Qureshi et al. (2001) reported that the amount of water utilized by cane plant has a linear relationship to total dry matter produced. A favorable soil water condition during cane growth also has a significant effect on the yield and quality response of sugar cane to nitrogen fertilization. Bhatti et al. (1986). According to Taha et al. (2003) meeting the nutrient and water requirements of sugar cane effectively makes the crop flourish and yield profitably. Yahaya et al. (2010) indicated that nitrogen application (120 and $240 \mathrm{~kg} / \mathrm{ha}$ ) significantly increase the yield of cane, the highest dose of nitrogen (240 $\mathrm{kg} / \mathrm{ha}$ ) resulted in poor cane quality. On the same trend Shahrzad and Kamla (2014) provided that increasing applied N levels from 140 up to $200 \mathrm{Kg} \mathrm{N} / \mathrm{fed}$ significantly increased stalk length, stalk diameter, stalk yield, sugar yields, TSS (\%), sucrose (\%) and purity (\%).
On the contrary Andressa et al. (2016) reported that the technological variables Brix $\%$, Pol $\%$ juice, Purity $\%$ and total recoverable sugar were altered by the application of nitrogen doses, with significant reductions at the dose of $200 \mathrm{~kg} \mathrm{~N} / \mathrm{ha}^{-1}$.

The role of sugar cane variety is considered the main factor in governing the expected sugar yield. It is well to known that, the commercial variety G.T.54-9(C9) occupies most of sugar cane area in Egypt. Recently, Sugar Crops Research Institute produced some promising varieties of sugar cane among them G.2003-47, G.99-103, G.2004-27, and G.2010-7. Many studies were Designed to select among the produced varieties in yield and its components, as well as, juice quality parameters among them Rizk, et al. (2004), El-Shafai \&Ismail (2006), ElLabbody et al. (2011), Mohamed et al. (2012), Yousif et al. (2015), Ahmed et al. (2016) and Ahmed (2017).

The objectives of this research were to determine the effect of nitrogen fertilizer levels on sugar yield, yield components and sugar quality of five sugar cane varieties under Middle Egypt conditions.

\section{MATERIALS AND METHODS}

Two field experiments were conducted at Mallawy Agricultural Research Station, Agricultural Research Center, Minia Governorate, Egypt during the two successive growing seasons of 2016/ 17 (plant crop) and 2017/ 18 (first ratoon), to study the effect of nitrogen fertilizer levels on sugar yield, yield components and sugar quality of five sugar cane varieties under Middle Egypt conditions.

A randomized complete block with three replications arranged in split- plot design was used, five sugar cane varieties $\left(\mathrm{V}_{1}\right.$ : G.T.54-9(C9) as a commercial variety, $\mathrm{V}_{2}$ : G.2003-47, $\mathrm{V}_{3}$ : G.99-103, $\mathrm{V}_{4}$ : G.2004-27 and $\mathrm{V}_{5}$ : G.2010-7 as a promising varieties) were allocated to the main plots, while three $\mathrm{N}$-fertilizer levels treatments $\left(\mathrm{N}_{1}: 160 \mathrm{~kg} / \mathrm{fed}, \mathrm{N}_{2}: 190 \mathrm{~kg} / \mathrm{fed}\right.$ and $\left.\mathrm{N}_{3}: 220 \mathrm{~kg} / \mathrm{fed}\right)$ were randomly distributed in the sub- plots, $\mathrm{N}$-fertilizer levels 
were added in two equal doses the $1^{\text {st }}$ one after 60 days from planting and the $2^{\text {nd }}$ after 30 days later. Before planting soil samples were randomly taken from the experimental site at a depth of 0 to $30 \mathrm{~cm}$ and prepared for physical and chemical analysis (Table 1) according to standard methods by Ankerman and large (1974). Plot area was $35 \mathrm{~m}^{2}$ containing five ridges at seven meters length and one meter width. The dry method of planting was adopted using two ridges of cane cuttings. The planting was in the $2^{\text {nd }}$ week of March, 2016, while harvest was done in the $3^{\text {rd }}$ week of March 2017 and 2018 in the $1^{\text {st }}$ and $2^{\text {nd }}$ seasons. All the agronomic practices for growing sugar cane were carried out as recommended by the Sugar Crops Research Institute in the region.

Data recorded

\section{I.Growth characters:}

At harvest, five plants from each sub-plot were taken at random to determine the following data:

1- Stalk height $(\mathrm{cm})$ was measured from soil surface to the top point of visible dewlap.

2- Stalk diameter $(\mathrm{cm})$ was measured at the middle part of stalk.

3- Millable cane yield (ton/fed) cane stalks of each plot were off cleaned from trash, weighed and cane yield was calculated.

II. Juice quality:

1- Purity (\%) was calculated using the following formula according to Singh and Singh (1998)

Table 1. Some physical and chemical analysis of soil at the experimental soil at $30 \mathrm{~cm}$ depth in $2016 / 2017$ and 2017/2018 seasons.

\begin{tabular}{|c|c|c|c|c|c|c|}
\hline \multicolumn{7}{|c|}{ Physical properties } \\
\hline seasons & \multicolumn{2}{|r|}{ Sand\% } & \multicolumn{2}{|c|}{ Silt $\%$} & \multicolumn{2}{|r|}{ Clay\% } \\
\hline $1 \mathrm{st}$ & \multicolumn{2}{|r|}{9.63} & \multicolumn{2}{|c|}{61.87} & \multicolumn{2}{|r|}{28.50} \\
\hline \multirow[t]{2}{*}{$2^{\text {nd }}$} & & 7.52 & & & & 30.35 \\
\hline & \multicolumn{2}{|r|}{ Texture grade } & \multicolumn{3}{|c|}{ Clay loam } & \\
\hline \multicolumn{7}{|c|}{ chemical properties } \\
\hline seasons & Total N\% & Available ppm & Available $\%$ & $\mathrm{CACO}_{3} \%$ & $\mathrm{PH}$ & $\mathrm{EC}(\mathrm{m} \mathrm{mhos} / \mathrm{cm})$ \\
\hline 1 st & 0.45 & 11.25 & 0.081 & 2.06 & 7.65 & 1.11 \\
\hline $2^{\text {nd }}$ & 0.43 & 12.16 & 0.085 & 2.08 & 7.45 & 1.08 \\
\hline
\end{tabular}

The obtained data of the two investigated seasons were computed and statistically analyzed for testing the significance of the studied factors and their interactions by LSD test according to Steel and Torrie (1981).

\section{RESULTS AND DISCUSSION}

\section{Growth characters:}

Data presented in Table 2 show the effect of sugar cane varieties and $\mathrm{N}$-fertilizer levels as well as their interactions on stalk height, stalk diameter $(\mathrm{cm})$ and millable cane yield ton/fed in both seasons.

Sugar cane varieties differed significantly in stalk height, diameter $(\mathrm{cm})$ and millable cane yield ton/fed in both seasons. G.99-103 variety $\left(\mathrm{v}_{3}\right)$ produced the highest values of stalk height $(294.00,270.22$ and $282.11 \mathrm{~cm}$.), stalk diameter $(3.42,3.32$ and $3.37 \mathrm{~cm}$.) and millable cane yield $(60.10,58.70$ and 59.40 ton/fed) in plant cane, first ratoon crops and comb., respectively. While, the lowest values were obtained by G.2004-27( $\left.\mathrm{v}_{4}\right)(236.11,223.53$,
Juice purity (\%) = sucrose (\%) / TSS (\%) X100.

2- Total soluble solids (TSS \%) was measured by hand refractometer according to the methods outlined in the A.O.A.C. (1995).

3- Sucrose (\%) was determined by Digital Automatic Polarimeter A.O.A.C. (1995).

III. Sugar recovery (\%) and Sugar yield(ton/fed):

A sample of 25 plants from each sub-plot were chosen immediately after harvest, cleaned and crushed through mill and juice was analyzed to determine the following data:

1- Sugar recovery (\%) was calculated according to the following formula described by Yadav and Sharma (1980).

Sugar recovery $(\%)=[$ sucrose $\%-0.4($ TSS $\%$ - sucrose $\%)] \times 0.73$. Where:

$0.4=$ each pound of non-sucrose solids in the juice will retain 0.4 of a pound of sucrose as outlined by Hebert (1973).

$0.73=$ denote a correction factor for actual milling condition in factories that depends on the overall mean fiber percentage cane during processing as outlined by Mathur (1997).

2- Sugar yield (ton /fed) was calculated according to the following formula described by Mathur (1997).

Sugar yield(ton/fed) $=$ cane yield $($ ton/fed)XSugar recovery $(\%)$.

$229.82 \mathrm{~cm}, \quad 2.68,2.52,2.60 \mathrm{~cm}, \quad 39.60$ and 39.22 , $39.41 \mathrm{ton} / \mathrm{fed}$ ) for all previous traits in plant cane, first ratoon crops and comb., respectively. This result may be due to the genetic differences among varieties in their ability of the formation of internodes and determination of their length and diameter. These results are in according with those obtained by El-Shafai and Ismail (2006), ElLabbody et al. (2011), Mohamed et al. (2012) and Yousif et al. (2015).

Nitrogen fertilization levels significantly effect on stalk height, diameter $(\mathrm{cm})$ and millable cane yield ton/fed in both seasons. Increasing the applied $\mathrm{N}$ doses from 160 up to $220 \mathrm{Kg}$. N / fed increasing stalk height, diameter $(\mathrm{cm})$ and millable cane yield ton/fed, gradually. These results are in agreement with Nassar et al. (2005) and Vinicius et al. (2018).

The interaction between sugar cane varieties and nitrogen levels effects on all previous traits were not significant in plant cane and first ratoon crops. 
Table 2. Stalk height, Stalk diameter (cm.) and millable cane yield ton/fed of five sugar cane varieties as affected by three $\mathrm{N}$-fertilizer levels and their interactions during 2016/2017 and 2017/2018 season.

\begin{tabular}{|c|c|c|c|c|c|c|c|c|c|c|}
\hline \multirow{2}{*}{\multicolumn{2}{|c|}{$\begin{array}{l}\text { Characters } \\
\text { Treatments }\end{array}$}} & \multicolumn{3}{|c|}{ Stalk height(cm) } & \multicolumn{3}{|c|}{ Stalk diameter(cm) } & \multicolumn{3}{|c|}{ Millable cane yield ton/fed } \\
\hline & & 1st & 2nd & Comb. & 1st & 2nd & Comb. & 1st & 2nd & Comb. \\
\hline \multirow{5}{*}{ A:Variaties } & V1 & 275.00 & 254.37 & 264.68 & 2.97 & 2.92 & 2.94 & 48.10 & 46.67 & 47.38 \\
\hline & V2 & 260.00 & 243.62 & 251.81 & 2.77 & 2.68 & 2.72 & 46.40 & 45.93 & 46.16 \\
\hline & V3 & 294.00 & 270.22 & 282.11 & 3.42 & 3.32 & 3.37 & 60.10 & 58.70 & 59.40 \\
\hline & V4 & 236.11 & 223.53 & 229.82 & 2.68 & 2.52 & 2.60 & 39.60 & 39.22 & 39.41 \\
\hline & V5 & 258.33 & 238.98 & 248.66 & 2.71 & 2.63 & 2.67 & $\leqslant 0.1$. & 44.32 & 44.71 \\
\hline \multirow{2}{*}{$\begin{array}{l}\text { F-test } \\
\text { LSD at }_{0.05}\end{array}$} & & ** & ** & ** & $* *$ & $* *$ & $* *$ & $* *$ & $* *$ & ** \\
\hline & & 5.92 & 2.47 & 7.56 & 0.08 & 0.13 & 0.08 & 0.87 & 0.76 & 0.63 \\
\hline \multirow{3}{*}{$\begin{array}{l}\text { B:N-levels } \\
\text { Kg/fed }\end{array}$} & N1 & 239.47 & 219.75 & 229.61 & 2.72 & 2.57 & 2.65 & 45.84 & 44.80 & 45.32 \\
\hline & N2 & 267.60 & 249.07 & 258.33 & 2.93 & 2.84 & 2.89 & 47.58 & 46.60 & 47.09 \\
\hline & $\mathrm{N} 3$ & 287.00 & 269.62 & 278.31 & 3.07 & 3.03 & 3.05 & 50.16 & 49.50 & 49.83 \\
\hline \multirow{2}{*}{\multicolumn{2}{|c|}{$\begin{array}{l}\text { F-test } \\
\text { LSD at } \\
L_{0.05}\end{array}$}} & $* *$ & $* *$ & $* *$ & $* *$ & $* *$ & $* *$ & $* *$ & $* *$ & $* *$ \\
\hline & & 4.59 & 1.92 & 5.85 & 0.06 & 0.10 & 0.06 & 0.67 & 0.59 & 0.49 \\
\hline \multirow{15}{*}{$\begin{array}{l}\text { C:Interaction } \\
\mathrm{A} \times \mathrm{B}\end{array}$} & $\mathrm{V} 1 \times \mathrm{N} 1$ & 245.00 & 224.60 & 234.80 & 2.73 & 2.60 & 2.67 & 45.30 & 44.09 & 44.69 \\
\hline & $\mathrm{V} 1 \times \mathrm{N} 2$ & 280.00 & 257.50 & 268.75 & 3.00 & 3.00 & 3.00 & 48.00 & 46.43 & 47.22 \\
\hline & $\mathrm{V} 1 \times \mathrm{N} 3$ & 300.00 & 281.00 & 290.50 & 3.17 & 3.17 & 3.17 & 51.00 & 49.49 & 50.24 \\
\hline & $\mathrm{V} 2 \times \mathrm{N} 1$ & 235.00 & 217.10 & 226.05 & 2.57 & 2.43 & 2.50 & 44.10 & 43.41 & 43.76 \\
\hline & $\mathrm{V} 2 \times \mathrm{N} 2$ & 266.67 & 248.83 & 257.75 & 2.80 & 2.70 & 2.75 & 45.90 & 45.76 & 45.83 \\
\hline & $\mathrm{V} 2 \times \mathrm{N} 2$ & 278.33 & 264.93 & 271.63 & 2.93 & 2.90 & 2.92 & 49.20 & 48.61 & 48.90 \\
\hline & $\mathrm{V} 3 \times \mathrm{N} 1$ & 270.67 & 244.33 & 257.50 & 3.20 & 3.07 & 3.13 & 58.80 & 56.99 & 57.90 \\
\hline & $\mathrm{V} 3 \times \mathrm{N} 2$ & 301.33 & 276.50 & 288.92 & 3.43 & 3.30 & 3.37 & 60.00 & 58.29 & 59.14 \\
\hline & $\mathrm{V} 3 \times \mathrm{N} 3$ & 310.00 & 289.83 & 299.92 & 3.63 & 3.60 & 3.62 & 61.50 & 60.81 & 61.15 \\
\hline & $\mathrm{V} 4 \times \mathrm{N} 1$ & 208.33 & 195.77 & 202.05 & 2.53 & 2.27 & 2.40 & 37.50 & 37.17 & 37.33 \\
\hline & $\mathrm{V} 4 \times \mathrm{N} 2$ & 233.33 & 222.50 & 227.92 & 2.70 & 2.60 & 2.65 & 39.30 & 38.93 & 39.11 \\
\hline & $\mathrm{V} 4 \times \mathrm{N} 3$ & 266.67 & 252.33 & 259.50 & 2.80 & 2.70 & 2.75 & 42.00 & 41.57 & 41.78 \\
\hline & $\mathrm{V} 5 \times \mathrm{N} 1$ & 238.33 & 216.93 & 227.63 & 2.57 & 2.50 & 2.53 & 43.50 & 42.33 & 42.91 \\
\hline & $\mathrm{V} 5 \times \mathrm{N} 2$ & 256.67 & 240.00 & 248.33 & 2.73 & 2.60 & 2.67 & 44.70 & 43.62 & 44.16 \\
\hline & $\mathrm{V} 5 \times \mathrm{N} 3$ & 280.00 & 260.00 & 270.00 & 2.83 & 2.80 & 2.82 & 47.10 & 47.02 & 47.06 \\
\hline \multicolumn{2}{|l|}{$\overline{\text { F-test }}$} & * & $* *$ & NS & NS & NS & $\mathrm{NS}$ & NS & NS & NS \\
\hline \multirow{2}{*}{\multicolumn{2}{|c|}{$\frac{\text { LSD at }_{0.05}}{\mathrm{~V}:}$}} & 10.26 & 4.29 & - & - & - & - & - & - & - \\
\hline & & 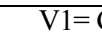 & 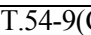 & $\overline{V 2}$ & $\overline{5}$ & & 33 , & $\sqrt{4-2}$ & .2 & \\
\hline
\end{tabular}

\section{Juice quality: $\quad$ tested sugar cane varieties in plant cane and first ratoon} Data presented in Table 3 pointed out that purity\%, crops, respectively.

$\mathrm{TSS} \%$ and sucrose $\%$ were differed significantly by the

Table 3. Purity\%, TSS\% and Sucrose \% of five sugar cane varieties as affected by three N- fertilizer levels and their interactions during 2016/2017 and 2017/2018 seasons

\begin{tabular}{|c|c|c|c|c|c|c|c|c|c|c|}
\hline \multirow{2}{*}{ Treatments } & \multirow[t]{2}{*}{ Characters } & \multicolumn{3}{|c|}{ Purity\% } & \multicolumn{3}{|c|}{ TSS\% } & \multicolumn{3}{|c|}{ Sucrose \% } \\
\hline & & 1st & 2nd & Comb. & 1st & 2nd & Comb. & 1st & 2nd & Comb. \\
\hline \multirow{5}{*}{ A:Variaties } & V1 & 83.00 & 82.71 & 82.85 & 21.09 & 20.86 & 20.97 & 17.48 & 17.23 & 17.36 \\
\hline & V2 & 76.69 & 77.85 & 77.27 & 20.54 & 20.34 & 20.44 & 15.74 & 15.80 & 15.77 \\
\hline & V3 & 79.76 & 80.23 & 80.00 & 19.31 & 19.19 & 19.25 & 15.40 & 15.39 & 15.39 \\
\hline & V4 & 76.76 & 77.46 & 77.11 & 21.11 & 20.98 & 21.04 & 16.19 & 16.23 & 16.21 \\
\hline & V5 & 76.45 & 76.03 & 76.24 & 20.67 & 20.80 & 20.73 & 15.76 & 15.78 & 15.77 \\
\hline \multirow{2}{*}{$\begin{array}{l}\text { F-test } \\
\text { LSD at } t_{0.05}\end{array}$} & & $* *$ & $* *$ & $* *$ & $* *$ & $* *$ & $* *$ & $* *$ & $* *$ & $* *$ \\
\hline & & 1.13 & 3.1 & 1.56 & 0.13 & 0.65 & 0.31 & 0.17 & 0.31 & 0.18 \\
\hline \multirow{3}{*}{$\begin{array}{l}\text { B:N-levels } \\
\mathrm{Kg} / \text { fed }\end{array}$} & N1 & 80.70 & 79.55 & 80.12 & 19.77 & 19.74 & 19.76 & 15.95 & 15.70 & 15.83 \\
\hline & N2 & 80.20 & 80.31 & 80.26 & 20.64 & 20.56 & 20.60 & 16.54 & 16.49 & 16.51 \\
\hline & N3 & 74.70 & 76.70 & 75.70 & 21.22 & 21.00 & 21.11 & 15.85 & 16.07 & 15.96 \\
\hline \multirow{2}{*}{$\begin{array}{l}\text { F-test } \\
\text { LSD at }{ }_{0.05}\end{array}$} & & $* *$ & * & $* *$ & $* *$ & $* *$ & $* *$ & $* *$ & $* *$ & $* *$ \\
\hline & & 0.88 & 2.40 & 1.21 & 0.10 & 0.51 & 0.24 & 0.13 & 0.24 & 0.14 \\
\hline \multirow{15}{*}{$\begin{array}{l}\mathrm{C}: \text { Interaction } \\
\mathrm{A} \times \mathrm{B}\end{array}$} & $\mathrm{V} 1 \times \mathrm{N} 1$ & 86.57 & 83.17 & 84.87 & 20.10 & 20.20 & 20.15 & 17.40 & 16.80 & 17.10 \\
\hline & $\mathrm{V} 1 \times \mathrm{N} 2$ & 83.81 & 84.73 & 84.27 & 21.20 & 20.50 & 20.85 & 17.77 & 17.37 & 17.57 \\
\hline & $\mathrm{V} 1 \times \mathrm{N} 3$ & 78.60 & 80.23 & 79.42 & 21.97 & 21.87 & 21.92 & 17.27 & 17.53 & 17.40 \\
\hline & $\mathrm{V} 2 \times \mathrm{N} 1$ & 77.99 & 77.28 & 77.63 & 20.13 & 20.10 & 20.12 & 15.70 & 15.53 & 15.62 \\
\hline & $\mathrm{V} 2 \times \mathrm{N} 2$ & 80.10 & 79.95 & 80.03 & 20.43 & 20.40 & 20.42 & 16.37 & 16.30 & 16.33 \\
\hline & $\mathrm{V} 2 \times \mathrm{N} 2$ & 72.00 & 76.33 & 74.16 & 21.07 & 20.53 & 20.80 & 15.17 & 15.57 & 15.37 \\
\hline & $\mathrm{V} 3 \times \mathrm{N} 1$ & 79.58 & 78.28 & 79.21 & 19.10 & 19.00 & 19.05 & 15.2 & 14.97 & 15.08 \\
\hline & $\mathrm{V} 3 \times \mathrm{N} 2$ & 82.71 & 82.96 & 82.84 & 19.27 & 19.17 & 19.22 & 15.93 & 15.90 & 15.92 \\
\hline & $\mathrm{V} 3 \times \mathrm{N} 3$ & 77.00 & 78.88 & 77.94 & 19.57 & 19.40 & 19.48 & 15.07 & 15.30 & 15.18 \\
\hline & $\mathrm{V} 4 \times \mathrm{N} 1$ & 77.49 & 78.12 & 77.80 & 20.43 & 20.10 & 20.27 & 15.83 & 15.70 & 15.77 \\
\hline & $\mathrm{V} 4 \times \mathrm{N} 2$ & 79.78 & 78.90 & 79.34 & 20.93 & 21.10 & 21.02 & 16.70 & 16.63 & 16.67 \\
\hline & $\mathrm{V} 4 \times \mathrm{N} 3$ & 72.99 & 75.35 & 74.17 & 21.97 & 21.73 & 21.85 & 16.03 & 16.37 & 16.20 \\
\hline & $\mathrm{V} 5 \times \mathrm{N} 1$ & 81.85 & 80.33 & 81.09 & 19.10 & 19.30 & 19.20 & 15.63 & 15.50 & 15.57 \\
\hline & $\mathrm{V} 5 \times \mathrm{N} 2$ & 74.57 & 75.03 & 74.80 & 21.37 & 21.63 & 21.50 & 15.93 & 16.23 & 16.08 \\
\hline & $\mathrm{V} 5 \times \mathrm{N} 3$ & 72.91 & 72.72 & 72.82 & 21.53 & 21.47 & 21.50 & 15.70 & 15.60 & 15.65 \\
\hline \multirow{2}{*}{\multicolumn{2}{|c|}{$\begin{array}{l}\text { F-test } \\
\text { LSD at } \\
0.05\end{array}$}} & $* *$ & NS & $* *$ & $* *$ & NS & $* *$ & $* *$ & NS & $*$ \\
\hline & & 1.96 & - & 2.71 & 0.23 & 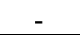 & 0.53 & 0.30 & - & 0.32 \\
\hline
\end{tabular}

Moreover, $\left(\mathrm{V}_{1}\right)$ G.T.54-9 (C9) sugar cane variety surpassed all tested varieties in purity $\%$ of $(83.00 \%$, $82.71 \%$ and $82.85 \%)$ and sucrose $\%$ of $(17.48 \%, 17.23 \%$ and $17.30 \%$ ) in plant cane and first ratoon crops and comb., respectively. while $\left(\mathrm{V}_{4}\right)$ G.2004-27variety recorded the best TSS\% of $(21.11 \%, 20.98 \%)$ in both seasons, respectively. This result may be due to the genetic differences among the tested varieties and the surrounding 
environmental conditios prevailing during maturity development of purity $\%$, TSS $\%$ and sucrose $\%$. These results are in agreement with that reported by Rizk et al. (2004), El- Labbody et al. (2011), Mohamed et al. (2012) and Ahmed et al. (2016).

Concerning the effect of $\mathrm{N}$ - fertilization levels, it was concluded that all Juice quality traits were significantly affected by $\mathrm{N}$ - fertilization levels in the plant cane and $1^{\text {st }}$ ratoon crops, respectively. the application of $190 \mathrm{~kg}-\mathrm{N} / \mathrm{fed}$ resulted the best values for purity $\%$ of $80.31 \%$ and sucrose $\%$ of (16.54 and16.49) in the second and both seasons, respectively, while $160 \mathrm{~kg}-\mathrm{N} / \mathrm{fed}$ treatment obtained the best purity $\%$ of $80.71 \%$ in the plant cane crop. Moreover, the application of $220 \mathrm{~kg}-\mathrm{N} / \mathrm{fed}$ occupied the best values for TSS\% of (21.22 and 21.00) in the plant cane and $1^{\text {st }}$ ratoon crops, respectively. These results were the same trend with obtained by those Nassar et al. (2005), Shahrzad and Kamla (2014), Yousif et al. (2015), Andressa et al.(2016) and Vinicius et al. (2018).

The interaction between sugar cane varieties and nitrogen levels effects on all Juice quality traits were significantly differed significantly in plant crop only. $\left(\mathrm{V}_{1}\right)$ G.T.54-9(C9) sugar cane variety with $160 \mathrm{~kg}-\mathrm{N} / \mathrm{fed}$ surpassed all tested varieties in purity $\%$ of $(86.57 \%)$, and with $190 \mathrm{~kg}-\mathrm{N} / \mathrm{fed}$ obtained the highest value for sucrose $\%$ of $17.77 \%$, moreover, with $220 \mathrm{~kg}-\mathrm{N} /$ fed cleared highly TSS $\%$ value of $21.97 \%$.

III. Sugar recovery (\%) and Sugar yield (ton/fed):

The obtained results in Table 4 revealed that the tested varieties significantly differed in sugar recovery $(\%)$ and sugar yield (ton/fed) in the plant cane and $1^{\text {st }}$ ratoon crops. The best sugar recovery (\%) of $11.70 \%, 11.52 \%$ and $11.61 \%$ were recorded by $\left(\mathrm{V}_{1}\right) \mathrm{C} 9$ variety in the plant cane, $1^{\text {st }}$ ratoon crops and comb. respectively, while sugar cane $\left(V_{3}\right)$ G.99-103 variety surpassed all tested varieties for sugar yield (ton/fed), which recorded the highest mean values of $\left(6.07,5.94\right.$ and 6.01 ton/fed) in the plant cane, $1^{\text {st }}$ ratoon crops and comb., respectively. on contrary $\left(\mathrm{V}_{4}\right)$ G.2004-27variety had the lowest values (4.11, 4.1 r and $4.11 \mathrm{ton} / \mathrm{fed}$,) in both seasons and comb., respectively. This result may be due to variation in genetics structure among tested sugar cane varieties. These results are in harmony with those of Rizk et al. (2004), El-Shafai and Ismail (2006), El- Labbody et al. (2011), Mohamed et al. (2012) and Ahmed et al. (2016) they found that statistical differences in sugar yield and sugar recovery $\%$ were recorded among the studies varieties.

Table 4. Sugar recovery\% and Sugar yield (ton/fed) of five sugar cane varieties as affected by three $\mathrm{N}$-fertilizer levels and their interactions during 2016/2017 and 2017/2018 season.

\begin{tabular}{|c|c|c|c|c|c|c|c|}
\hline \multirow{2}{*}{\multicolumn{2}{|c|}{\begin{tabular}{l}
\multicolumn{2}{c}{ Characters } \\
Treatments
\end{tabular}}} & \multicolumn{3}{|c|}{ Sugar recovery\% } & \multicolumn{3}{|c|}{ Sugar yield (ton/fed) } \\
\hline & & 1st & 2nd & Comb. & 1st & 2nd & Comb. \\
\hline \multirow{5}{*}{ A:Variaties } & V1 & 11.70 & 11.52 & 11.61 & 5.62 & 5.38 & 5.50 \\
\hline & V2 & 10.09 & 10.21 & 10.15 & 4.67 & 4.69 & 4.68 \\
\hline & V3 & 10.10 & 10.12 & 10.11 & 6.07 & 5.94 & 6.01 \\
\hline & V4 & 10.38 & 10.46 & 10.42 & 4.11 & $4.1 r$ & 4. 11 \\
\hline & V5 & 10.54 & 10.05 & 10.30 & 4.76 & 4.45 & 4.61 \\
\hline \multicolumn{2}{|l|}{ F-test } & $* *$ & $* *$ & $* *$ & $* *$ & $* *$ & $* *$ \\
\hline \multicolumn{2}{|l|}{$\underline{\mathrm{LSD}}_{\mathrm{at}} \mathrm{t}_{0.05}$} & 0.67 & 0.39 & 0.38 & 0.34 & 0.23 & 0.20 \\
\hline \multirow{3}{*}{$\begin{array}{l}\mathrm{B}: \mathrm{N}-\mathrm{levels} \\
\mathrm{Kg} / \text { fed }\end{array}$} & N1 & 10.53 & 10.28 & 10.41 & 4.82 & 4.59 & 4.71 \\
\hline & $\mathrm{N} 2$ & 10.88 & 10.85 & 10.86 & 5.17 & 5.05 & 5.11 \\
\hline & N3 & 10.28 & 10.29 & 10.29 & 5.15 & 5.09 & 5.12 \\
\hline \multirow{2}{*}{\multicolumn{2}{|c|}{ F-test }} & NS & $* *$ & $* *$ & $*$ & $* *$ & $* *$ \\
\hline & & - & 0.30 & 0.30 & 0.26 & 0.18 & 0.16 \\
\hline \multirow{15}{*}{$\begin{array}{l}\mathrm{C}: \text { Interaction } \\
\mathrm{A} \times \mathrm{B}\end{array}$} & $\mathrm{V} 1 \times \mathrm{N} 1$ & 11.91 & 11.27 & 11.59 & 5.40 & 4.97 & 5.18 \\
\hline & $\mathrm{V} 1 \times \mathrm{N} 2$ & 11.97 & 11.76 & 11.86 & 5.74 & 5.46 & 5.60 \\
\hline & $\mathrm{V} 1 \times \mathrm{N} 3$ & 11.23 & 11.53 & 11.38 & 5.73 & 5.71 & 5.72 \\
\hline & $\mathrm{V} 2 \times \mathrm{N} 1$ & 10.17 & 10.01 & 10.09 & 4.49 & 4.34 & 4.41 \\
\hline & $\mathrm{V} 2 \times \mathrm{N} 2$ & 10.76 & 10.70 & 10.73 & 4.94 & 4.90 & 4.92 \\
\hline & $\mathrm{V} 2 \times \mathrm{N} 2$ & 9.35 & 9.91 & 9.63 & 4.60 & 4.82 & 4.71 \\
\hline & $\mathrm{V} 3 \times \mathrm{N} 1$ & 9.96 & 9.75 & 9.85 & 5.86 & 5.56 & 5.71 \\
\hline & $\mathrm{V} 3 \times \mathrm{N} 2$ & 10.66 & 10.65 & 10.66 & 6.39 & 6.21 & 6.30 \\
\hline & $\mathrm{V} 3 \times \mathrm{N} 3$ & 9.68 & 9.97 & 9.83 & 5.96 & 6.07 & 6.01 \\
\hline & $\mathrm{V} 4 \times \mathrm{N} 1$ & 10.22 & 10.18 & 10.20 & 3.83 & 3.78 & 3.81 \\
\hline & $\mathrm{V} 4 \times \mathrm{N} 2$ & 10.95 & 10.84 & 10.90 & 4.30 & 4.22 & 4.26 \\
\hline & $\mathrm{V} 4 \times \mathrm{N} 3$ & 9.97 & 10.38 & 10.18 & 4.19 & 4.32 & 4.25 \\
\hline & $\mathrm{V} 5 \times \mathrm{N} 1$ & 10.40 & 10.21 & 10.30 & 4.52 & 4.32 & 4.42 \\
\hline & $\mathrm{V} 5 \times \mathrm{N} 2$ & 10.04 & 10.27 & 10.16 & 4.49 & 4.48 & 4.49 \\
\hline & $\mathrm{V} 5 \times \mathrm{N} 3$ & 11.18 & 9.67 & 10.43 & 5.27 & 4.55 & 4.91 \\
\hline \multicolumn{2}{|l|}{ F-test } & $\mathrm{NS}$ & $\mathrm{NS}$ & $\mathrm{NS}$ & NS & NS & $* *$ \\
\hline \multirow{2}{*}{\multicolumn{2}{|c|}{$\frac{\mathrm{LSD} \mathrm{at}_{0.05}}{\mathrm{~V} \cdot}$}} & - & - & - & - & - & 0.35 \\
\hline & & T.T.54- & $\mathrm{V} 2=\mathrm{C}$ & $\mathrm{V} 3=$ & , V4= & $27, \mathrm{~V}$ & \\
\hline
\end{tabular}

Regarding the effect of $\mathrm{N}$ - fertilization levels, it was concluded that sugar recovery (\%) and sugar yield (ton/fed) were significantly affected by $\mathrm{N}$ - fertilization levels in the plant cane, $1^{\text {st }}$ ratoon crops and comb. respectively. The application of $190 \mathrm{~kg}-\mathrm{N} /$ fed resulted the best values for sugar recovery (\%) of $10.88 \%, 10.85 \%$ and $10.86 \%$ in both seasons and comb., respectively, as well as sugar yield of (5.17ton/fed) in the plant cane crop season. However $220 \mathrm{~kg}-\mathrm{N} /$ fed recorded the highest sugar yield of (5.09 and 5.12 ton/fed) in the $1^{\text {st }}$ ratoon crop and comb. respectively. This result may be due to the effect of $\mathrm{N}$ - fertilization on increasing stalk height, diameter $(\mathrm{cm})$ and millable cane yield ton/fed These results are in the line with that reported by El-Geddawy et al. (2003), ElGeddawy et al. (2005), Nassar et al. (2005), Shahrzad and Kamla (2014), Yousif et al. (2015) and Vinicius et al. (2018).

The interaction between sugar cane varieties and nitrogen levels effects on sugar recovery (\%) and sugar yield (ton/fed) traits was not significant effect in plant cane, first ratoon crops and comb. respectively. 


\section{REFERENCES}

Ahmed, A.Z. (2017). Response of three sugar cane varieties to phosphorus bio fertilization. Egypt J. Agron., 42(2):149-158.

Ahmed, A.Z.; El-Bakry, A. and Sakina, R. (2016). Assessment of the optimnm age for harvesting some promising sugar cane varieties.Minia, J. Agric. Res. \& Develop., 36(4):635-651.

Andressa, F.L. Rhein; Pincelli , R.P. ; Arantes, M.T. ; Dellabiglia W.J. ; Kolln, O.T. and Silva M.A. (2016). Technological quality and yield of sugar cane grown under nitrogen doses via subsurface drib fertigation.Eng.Agric. Ambi., 20(3):209-214.

Ankerman,D. and Large, L. (1974). Soil and plant analysis, ASL Agricultural laboratories. Inc.New York, USA.

A.O.A.C. (1995). Association of Official Analysis Chemists" Official Methods of Analysis". $16^{\text {th }}$ ed., A.O.A.C. International, D.C., USA.

Azzazy, N.B. and El-Geddawy, I.H. (2003). Effect of nitrogen fertilization on yield and quality of some sugar cane varieties under developed surface irrigation system. Egypt. j. Agric. Res., 81(3): 1137-1147.

Bhatti, H.M.; Ullah, M. H.; Khan, M.R. and Gill, M.R. (1986). Consumptive use of water for sugar cane crop. J. Agric. Res., 24(1): 59-60.

El-Geddawy, I.H.; Rizk, M.A. M.; Taha, M.G.A. and Osman, M.S.H. (2003). Effect of nitrogen fertilization on yield and yield components of sugar cane. Egypt. J.Agric. Res.,81(4):1657-1669.

El-Geddawy, I.H.; Rady, M.S.; Dawwam, H.A.; Hendawy, F.A. and Abo El-Ghait, R.A.M. (2005). Response of some sugar cane varieties, nitrogen and potassium application. Egypt J. Agric. Res., 83(2): 693-716.

EL-Labbody, A.H.S.A.; Osman, A.M.H. and Osman, M.S.H. (2011). Performance of two promising sugar cane varieties under different inter row spacing. J. Plant Prod. Mansoura Univ., 2(2):297305.

EL-Shafai, A.M.A. and Ismail, A.M.A. (2006). Effect of row spacing on yield and quality of some promising sugar cane varieties. Egypt. j., Appl. Sci. 21 (11):32-46.

Hebert, L.P. (1973). Testing of sugar cane varieties for milling quality. The Sugar J., 36 (4):8-12.

Mathur, R.B.L. (1997). " Handbook of Cane Technology". Oxford IBH publishing Co. PVT. LTD New Delhi. Calcutta.
Mohamed, K.M.; Elwan, A.M. and Tawfik, S.F. (2012). The effect of cultivars and harvest time on yield and quality of sugar cane. Minia, J. Agric. Res. \& Develop., 32(5):35-48.

Nassar, A.M.; El-Sagheir, K.S. and Ramadan, B.S.H. (2005). Effect of nitrogen levels on yield and juice quality of some sugar cane varieties. Egypt. J. Agric. Res., 83(2): 681-692.

Qureshi, M. E.; Wegener, M.K.; Harison, S.R. and Bristow, K.L. (2001) Economic evaluation of irrigation system for sugar cane in the Burdekin delta in North Queensland, Australia. Water Resource Management, WIT Press, Boston:47-57.

Rizk, T.Y.; El-Agroudy, M.H.; El-Geddawy, I.H. and Fergany, M.A. (2004). Effect of row spacing and cutting size on cane juice quality attributes. Egypt. j. Agric. Res., 82(1): 251-262.

Shahrzad, M.M. Neana; Kamla, A.E. and Abd El Hak (2014). Effect of irrigation regime and nitrogen fertilization levels on sugar cane yield and its components. Alex. Sci. Exchange J.,35. (4) 288294.

Singh,R.P.and Singh, P.P. (1998). Quality of juice and commercial sugar cane as influenced by crop cane. Indian J. Sugar, 48(1):21-28.

Steel, R.G.D. and Torrie, J.H. (1981). Principles and Procedures of Statistic, Abiometrical approach. $2^{\text {nd }}$ ed.by Mc Graw -Hill International Book Company, Singapore, 633.

Taha, E.M.; Ahmed, A.Z. and El-Sagheir, K.S. (2003). Response of four sugar cane varieties to potassium fertilizer. Egypt. J. Agric. Res., 81(1): 151-160.

Vinicius, S. G. S.; Mauro, W. O.; Vilma, M. F.; Terezinha, B. A. O.; Elaine, R. G.; Aleksandro, F. S. and Polyana A. S. M. (2018). Nutritional requirement of sugar cane varieties. j. agric.sci. 10(4): 361-369.

Yadav, R.L. and Sharma, R.K. (1980). Effect of nitrogen level and harvesting date on quality characteristics and yield of four sugar cane genotypes. Indian J. Agric.Sci.,(50):581-589.

Yahaya,M.S; Falaki, A.M.; Amans, E.B. and Busari, L D. (2010). Sugar cane yield and quality as influenced by nitrogen rates and irrigation frequency. Nigerian J. Res., and Prod., 17 (2):1-10.

Yousif, E.M.M.; Ibrahim, M.M.; El- Aref, A.O.K. and Ahmed, A.Z. (2015). Management of nitrogen fertilization for sugar cane on a sandy soil: 1 yield and its components. Egypt. J. Appl. Sci.30 (11):498-511.

Yousef, M.A., Taha, E.M. and Ahmad, A.Z. (2000). Influence of some cultural practices on yield and yield components of some sugar cane varieties. Egypt.J. Agric. Res., 78 (5): 1995-2008.

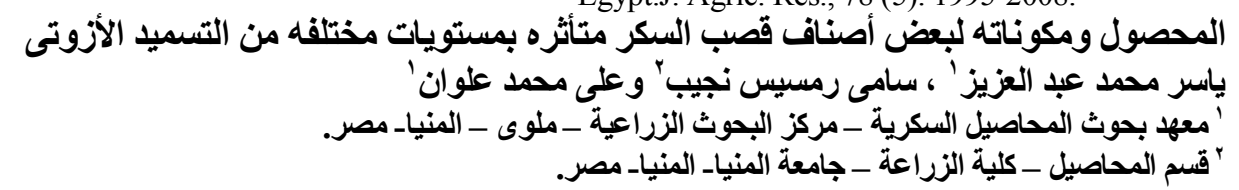

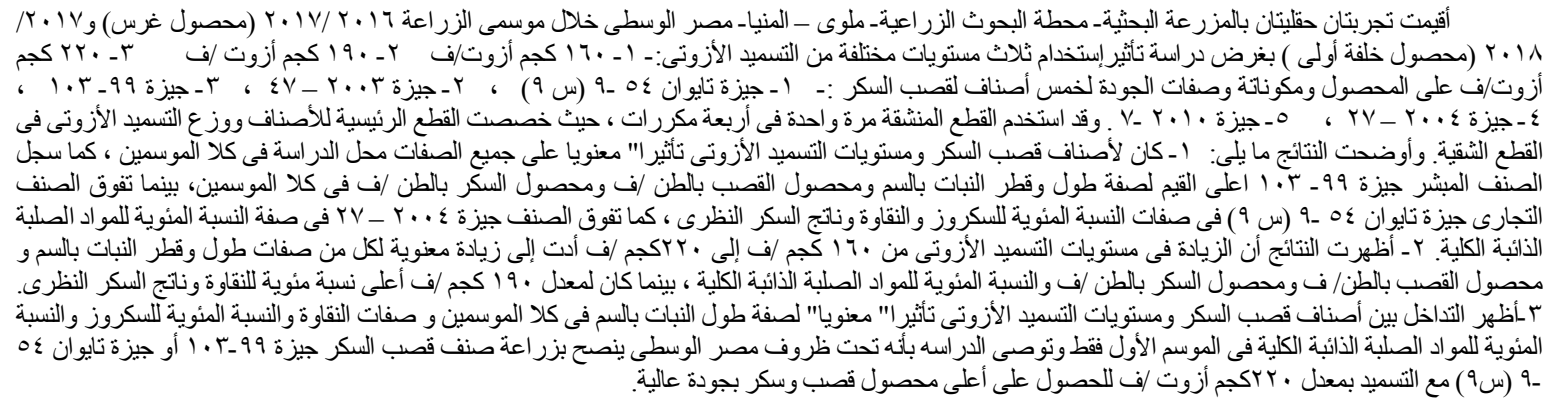

